\title{
A Mesh-Based Model for Prediction of Initial Tooth Movement
}

\author{
K. De Bondt ${ }^{1}$, A. Van Schepdael ${ }^{1}$, J. Vander Sloten ${ }^{1}$ \\ ${ }^{1}$ K.U.Leuven, Division of Biomechanics and Engineering Design, Belgium
}

\begin{abstract}
Orthodontics is making an evolution from a highly experience based treatment towards a computer assisted patient specific therapy. The development of models that can predict tooth movement therefore is a critical research topic. Many existing models, FEM as well as analytical, simplify the calculations by assuming that the tooth root can be approximated by a paraboloïd or an elliptical paraboloïd. Other studies state that these approximations are only allowed within a certain range for tipping movements. But when it comes to bodily movement the real root and the approximation can give very different FEM results. This paper presents a model that is based on an analytical approach using paraboloïds. The analytical approach is chosen to avoid FEM analysis and to limit calculation time. The developed model expands the applicability so that it can do the calculations for a mesh representation of the root. As a validation a set of elliptical paraboloïds is constructed and the results for the analytic and the mesh-based procedure are compared. This is done by comparing the forces necessary to cause a certain displacement or rotation of the root. Both methods produced the same results except for small deviations that can be assigned to the mesh accuracy and the accuracy of the numerical integration that is used within the analytical approach. This makes the presented model an interesting tool for the evaluation of the correctness of an approximating paraboloïd. It is also suitable for the calculation of initial tooth movement as part of a model for the simulation of orthodontic tooth movement.
\end{abstract}

Keywords - mesh, modeling, movement, prediction, tooth

\section{INTRODUCTION}

Orthodontics is still a highly experience-based treatment and a 'perfect dentition' is often reached through a process of trial and error. There is a tendency towards movement predictive modeling and simulation to avoid this trial and error procedure and to shorten the treatment time and cost. Therefore FEM models and analytical models have been built to get a clearer view of the movement determining factors. Many authors use a paraboloïd as an approximation of the tooth root, with its height and diameter as adaptable parameters [1], [2], [3]. Paraboloïds are not suited to describe all root geometries in an exact way. For this reason the elliptical paraboloïds are introduced as a common representation of the root geometry [4]. Few studies report on the accuracy of these paraboloïds to predict the movement of a real tooth. Some [5] state, based on FEM analysis, that for tipping movements the results only differ within a range of $10 \%$ but that the differences are much higher for bodily movement. As a contribution to this research topic, this paper will present a model that predicts the stress in the periodontal ligament for a triangle mesh of a tooth root and that derives the initial displacement of the root. The use of FEM analysis will be avoided and calculations will be based on analytical formulas to keep the calculation time low. This makes the model applicable as an evaluation tool to compare real root geometries to paraboloïd approximations, and as a model for initial tooth movement prediction.

\section{MATERIALS AND METHODS}

The developed model is based on the approach of Provatidis [1] and Van Schepdael [4]. Provatidis reports the calculation of the one-directional movement of a singlerooted tooth induced by a force applied in the Cre of the paraboloïd representation Figure 1. The paraboloïd geometry is described by two parameters, height and diameter. He assumes the PDL has a constant thickness $\delta=0.229 \mathrm{~mm}$, with Young's modulus $\mathrm{E}=0.68 \mathrm{MPa}$ and Poisson coefficient $v=0.49$ which makes the PDL nearly incompressible. In [4] the force system (F, M) applied at the tooth bracket is transformed to the apex of the root and a stiffness matrix $(\mathrm{K})$ of the tooth root is calculated. The initial displacement vector ' $u$ ' can then be found by the equation $\mathrm{F}=\mathrm{K} . \mathrm{u}$.

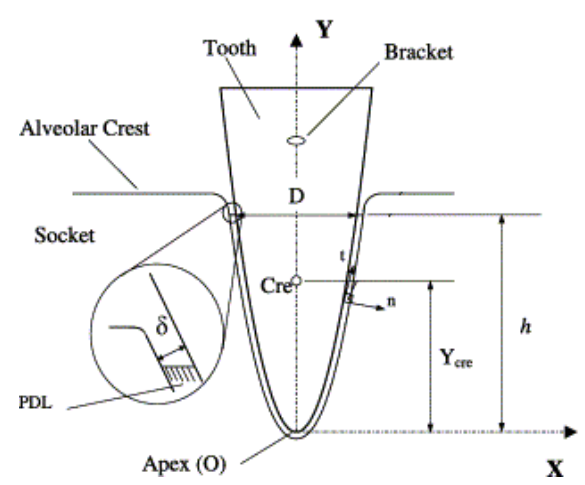

Figure 1: The geometry of the tooth[1] 
The model which introduced a combined calculation method for translation and rotation also makes use of elliptical paraboloïd geometries.

This adds an extra degree of freedom for the root geometry description, namely the eccentricity. The equation of a twodimensional ellipse with its centre at $(0,0)$ in polar coordinates $(r, \phi)$ is

$$
r^{2}=\frac{b^{2}}{1-e^{2} \cos ^{2} \phi}
$$

where $b$ is the short axis of the ellipse, and $e$ is its eccentricity. The relation between the short axis and the long axis is expressed by:

$$
b^{2}=a^{2} \cdot\left(1-e^{2}\right)
$$

where $a$ is the long axis length. In the case of an 'elliptic' paraboloid, where the long axis varies with the height $y$ :

$$
a^{2}=\frac{R^{2}}{h} y
$$

Where $R$ is the length of the long axis at $y=h$. The $y-$ coordinate for every point in the $\mathrm{xz}$-plane is then defined by:

$$
y=\frac{\left(1-e^{2} \cos \phi\right) \cdot 4 \cdot h \cdot r^{2}}{D^{2}\left(1-e^{2}\right)}
$$

In both the described analytical models the stiffness matrix is built starting from the strains defined in a local curvilinear system. By transforming the strains to the global axis and substituting them into Hooke's law, the stresses are calculated. Together with the normals to the surface the stresses define the tractions along the tooth surface. The stiffness matrix results from integrating the tractions along the tooth surface over the total root geometry. The idea of this study was to integrate the appropriate formulas over a triangle mesh instead of analytically described paraboloïd. In that way all existing types of root geometries could be simulated. To make this possible the formulas were adapted to be applicable for one arbitrary triangle. Also the integration boundaries were defined for one arbitrary triangle and the values of all individual triangles were added. To validate the used methods a comparison was made between the values resulting from the analytical model and those of the introduced mesh-based approach. Therefore an elliptical paraboloïd stl-set was created in Matlab $\odot$ defining a grid of $0.05 \mathrm{~mm} \times 0.05 \mathrm{~mm}$ in the $\mathrm{xz}-$ plane and calculating the appropriate y-coordinate (height) according to equation 4 for an elliptical paraboloï. The maximum allowed height for points in the defined grid was $15 \mathrm{~mm}$. All calculated y-coordinates higher than $15 \mathrm{~mm}$ were set to $15 \mathrm{~mm}$. The mesh was exported as an stl-file and adapted in Magics $\subset$ where the upper side, above $13 \mathrm{~mm}$, was cut. This was done to avoid the inaccuracies that showed up in the transition at $y=15 \mathrm{~mm}$. The resulting geometry was an accurate representation of the elliptical paraboloïd with height $13 \mathrm{~mm}$. The constructed meshes were simulated with the mesh-based model and the forces necessary to induce a displacement on this root geometry were calculated. Three different translations were induced, namely $u_{x}=2.10^{-4} \mathrm{~mm}, u_{y}=2.10^{-4} \mathrm{~mm}, u_{z}=2.10^{-4} \mathrm{~mm}$ and three different rotations $\theta_{x}=2.10^{-5} \mathrm{rad}, \theta_{y}=2.10^{-5} \mathrm{rad}, \theta_{z}$ $=2.10^{-5} \mathrm{rad}$. Secondly the results were compared to those of the elliptical paraboloïd model [4].

\section{RESUlts}

Table 1 Force necessary to cause a given displacement for elliptic paraboloïds with rising eccentricity

\begin{tabular}{cccccccc}
\hline eccentricity & 0 & 0.1 & 0.2 & 0.3 & 0.4 & 0.5 & 0.6 \\
\hline ux-Fx & 1.0725 & 1.066 & 1.0463 & 1.0129 & 0.9647 & 0.9 & 0.816 \\
uy-Fy & 0.1666 & 0.1656 & 0.1626 & 0.1575 & 0.1503 & 0.1406 & 0.1282 \\
uz-Fz & 1.0725 & 1.0738 & 1.078 & 1.0852 & 1.0958 & 1.1104 & 1.1301 \\
thx-Mx & 8.8603 & 8.8607 & 8.8625 & 8.8668 & 8.8759 & 8.8936 & 8.9262 \\
thy-My & 0.0393 & 0.039 & 0.0381 & 0.0367 & 0.0346 & 0.0319 & 0.0287 \\
thz-Mz & 8.8603 & 8.8063 & 8.6429 & 8.3652 & 7.9647 & 7.4272 & 6.73 \\
\hline
\end{tabular}

The forces necessary to cause a displacement $u_{x}=u_{y}=u_{z}=2.10^{-4} \mathrm{~mm}$ or a rotation $\theta_{x}=\theta_{y}=\theta_{z}=2.10^{-5}$ rad, were calculated for elliptical paraboloïd meshes with increasing eccentricity.

The long axis length at height $\mathrm{y}=13 \mathrm{~mm}$ was kept constant for all the geometries. The results are listed in Table 1. Due to the axisymmetric shape of the paraboloid (eccentricity $=0$ ) with the $y$-axis as the central axis, the forces necessary for a displacement in the $\mathrm{x}$ and $\mathrm{z}$-direction are equal. So are the moments $\mathrm{M}_{\mathrm{x}}$ and $\mathrm{M}_{\mathrm{z}}$. With ascending eccentricity (decreasing z-axis length a.k.a. short axis length) the needed force diminishes in the $x$-direction while it increases in the z-direction. This can be explained by the decrease in resisting surface for the $\mathrm{x}$-direction and the fact that the tooth is getting flatter when moving in the $\mathrm{z}$ direction. The change of the moments $M_{x}$ and $M_{z}$ can also be explained by these facts. In the y-direction the needed force $\mathrm{F}_{\mathrm{y}}$ decreases also due to the decrease in resisting surface. The moment around the y-axis was expected to increase, but instead there is a diminishing trend. An explanation can be found in the two effects that have an impact on the moment $\mathrm{M}_{\mathrm{y}}$. The first is the deviation from the perfect circle to more elliptic which makes the geometry more resistant to torsion moments. 


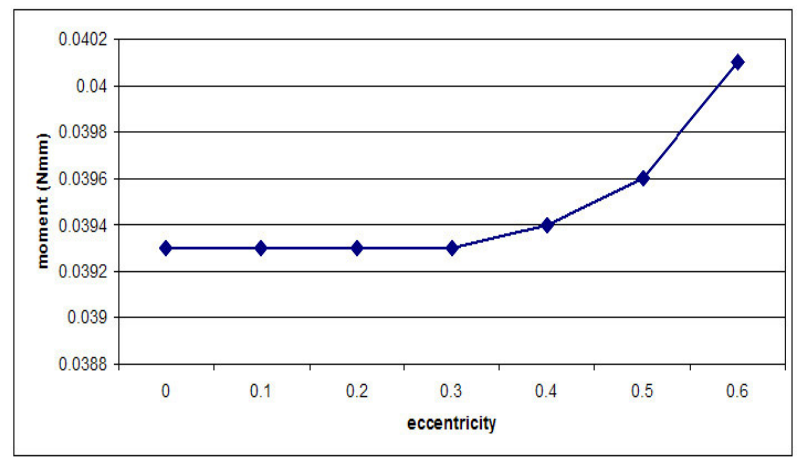

Figure 2 Forces Fy as calculated for a an elliptic root shape set with constant volume

The second is the decrease of total volume that must be rotated. To eliminate the second effect, a new set of elliptic paraboloïds was assessed where the total volume was remained constant. The moment $\mathrm{M}_{\mathrm{y}}$ of this geometry set showed the expected increase as can be seen in Figure 2, while the other moments and forces changed in the same way as previously described.

As an evaluation the necessary forces to cause a displacement were also calculated with the analytical model, for the same displacement and rotations. Results for the forces $F_{x}$ and $F_{z}$ are plotted within Figure 3. One can see that the trends tend to be the same: the analytical results (blue ' $x$ ') are approximately the same as the mesh-based results (red 'o'). Small disturbances can be accorded to the mesh accuracy and to the accuracy of the numerical integration in the analytical model. The overall calculation time was limited to a few seconds for a triangle mesh consisting of $\sim 40.000$ triangles.

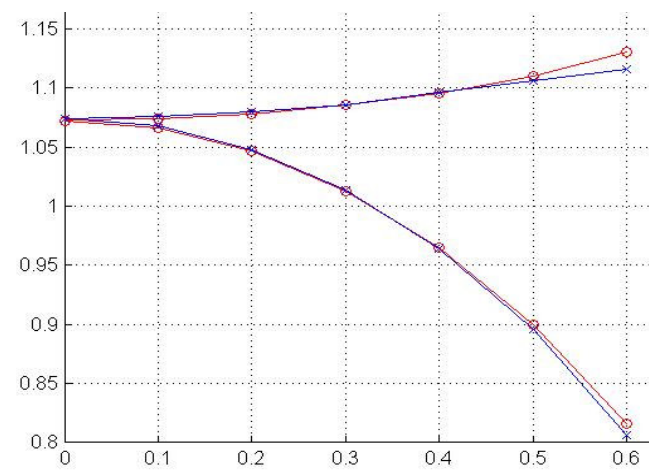

Figure 3 Forces Fx, Fz as calculated with analytical (blue,'x') and mesh based (red,'o')
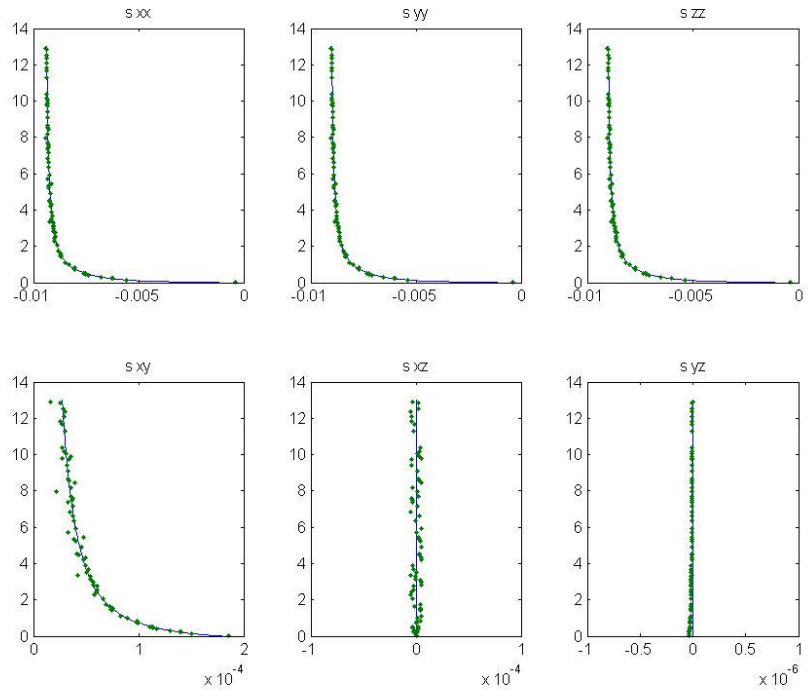

Figure 4 Stresses in the paraboloïd along the xy-plane, analytical results (blue,'line') compared to the mesh results (green,'dots')

A second evaluation was done based on the stresses reported by Provatidis [1], [6]. The stress patterns of the analytical model are compared to those of the mesh-based model in Figure 4. Again the presented model shows the same trends as the analytical approach.

\section{Discussion}

This study presents a model that calculates initial displacements from an applied force for tooth root geometries in a triangle mesh representation. Its concept was based on the work of Provatidis [1] and Van Schepdael [4], building a stiffness matrix for a paraboloïd tooth root. To validate the model, the forces necessary to induce a displacement were calculated for a mesh set of elliptical paraboloïds. This shows that the model is applicable for meshes of root geometries with results similar to the analytical model. One drawback for the applicability of the model that could be mentioned is its dependency of the mesh accuracy. But as the calculation time remains limited to a few seconds, it is possible to use large and accurate mesh representations.

The presented method is a contribution to the modeling of tooth movement that is needed to make orthodontics more predictive and less trial and error based. It can also be a valuable tool for evaluation of tooth root approximations. Most authors use a paraboloïd as a root representation, but as stated by Vollmer and Bourauel [5], results for bodily movement can differ significantly. To get a view on the 
correctness of an approximating paraboloïd the presented mesh-based model can be a valuable tool.

\section{ACKNOWLEDGMENT}

This research was funded by IWT-Vlaanderen and Materialise Dental NV (Belgium).

An Van Schepdael is a research assistant of the Research Foundation Flanders (FWO-Vlaanderen)

Clinical input from Prof Dr. G. Willems from the Departments of

Orthodontics and Forensic Odontology (Leuven)

\section{REFERENCES}

1. Christopher G.Provatidis (2001). An analytical model for stress analysis of a tooth in translation. Int. J. Eng Sci 39: 1361-1381.

2. Pedersen E., Andersen K., Gjessing P. E. (1990) Electronic determination of centres of rotation produced by orthodontic force systems, Eur. J. Orthod. 12: 272-280.
3. Burstone C.J., Pryputniewicz R.J., Bowley W.W (1978) Holographic measurement of tooth mobility in three dimensions, J Periodontal Res.: 283-294.

4. A.Van Schepdael, J.Vander Sloten (2008). [In Press] Effect of root form on stress patterns in the periodontal ligament Conference Proceedings CMBBE

5. D Vollmer, C.Bourauel, K.Maier, A Jäger, (1999) Determination of the centre of resistance in an upper human canine and idealized tooth model. Eur. J. Orthod. 21: 633-648.

6 Christopher G.Provatidis. Demetrios T.Venetsanos SDC (2007)

Estimation of the flexibilities of tooth support of an ellipsoidal shape

$2^{\text {nd }}$ International Conference on Experiments/Process/System

Modelling/Simulation \& Optimization

- $\quad$ Corresponding Author: Kris De Bondt

- Institute: K.U.Leuven

- $\quad$ Street: Celestijnenlaan 300C

- City: 3001 Heverlee

- Country: Belgium

- $\quad$ Email: kris.debondt@mech.kuleuven.be 